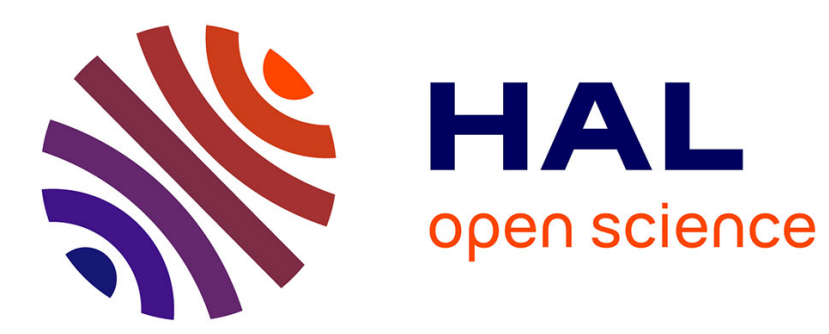

\title{
Deadline Scheduling with Priority for Client-Server Systems on the Grid
}

\author{
Eddy Caron, Pushpinder Kaur Chouhan, Frédéric Desprez
}

\section{To cite this version:}

Eddy Caron, Pushpinder Kaur Chouhan, Frédéric Desprez. Deadline Scheduling with Priority for Client-Server Systems on the Grid. [Research Report] RR-5335, LIP RR-2004-33, INRIA, LIP. 2004, pp.13. inria-00070666

\section{HAL Id: inria-00070666 https://hal.inria.fr/inria-00070666}

Submitted on 19 May 2006

HAL is a multi-disciplinary open access archive for the deposit and dissemination of scientific research documents, whether they are published or not. The documents may come from teaching and research institutions in France or abroad, or from public or private research centers.
L'archive ouverte pluridisciplinaire HAL, est destinée au dépôt et à la diffusion de documents scientifiques de niveau recherche, publiés ou non, émanant des établissements d'enseignement et de recherche français ou étrangers, des laboratoires publics ou privés. 


\section{Deadline Scheduling with Priority for Client-Server Systems on the Grid}

Eddy Caron, Pushpinder Kaur Chouhan, Frederic Desprez

$$
\mathbf{N}^{\circ} 5335
$$

Octobre 2004

THÈME 1 



\title{
Deadline Scheduling with Priority for Client-Server Systems on the Grid
}

\author{
Eddy Caron, Pushpinder Kaur Chouhan, Frederic Desprez \\ Thème 1 - Réseaux et systèmes \\ Projet ReMaP \\ Rapport de recherche $\mathrm{n}^{\circ} 5335$ - Octobre $2004-13$ pages
}

\begin{abstract}
We present algorithms for the scheduling sequential tasks on a Network Enabled Server (NES) environment. We have implemented the non-preemptive scheduling, since at the user level we cannot interrupt a running process and block it in order to allow a new process to run. This article is an extension of the paper: "A Study of Deadline Scheduling for Client-Server Systems on the Computational Grid" by Takefusa et al. We mainly discuss a deadline scheduling with priority strategy that is more appropriate for multi-client, multiserver case. Importance is first given to the task's priority and then the task is allocated to the server that can meet the task's deadline. This may cause that some already allocated tasks on the server miss their deadline. We augment the benefits of scheduling algorithms with load measurements (which is done with the use of a forecasting tool called FAST) and fallback mechanisms. The experimental results shows that the deadline scheduling with priority along with fallback mechanism can increase the overall number of tasks executed by the NES.
\end{abstract}

Key-words: Scheduling, Problem Solving Environment

This text is also available as a research report of the Laboratoire de l'Informatique du Parallélisme http: //www.ens-lyon.fr/LIP. 


\section{Ordonnancement à échéance avec priorité pour les systèmes client-serveur sur la Grille}

Résumé : Dans ce rapport, nous proposons des algorithmes pour l'ordonnancement de tâches séquentielles dans un environnement distribué de type NES (Network Enabled Server). Nous nous plaçons dans un cas non préemtif, il est donc impossible d'interrompre un processus en cours d'exécution pour en exécuter un autre. Ce rapport est une extension à l'article "A Study of Deadline Scheduling for Client-Server Systems on the Computational Grid" de Takefusa et al. une approche similaire à laquelle nous ajoutons un mécanisme de prédiction de performances. De plus, nous proposons d'inclure la notion de priorité qui est appropriée dans le cas des NES. L'ordonnanceur tient alors compte de la priorité de la tâche dans un premier temps, puis on vérifie son échéance (date à laquelle on souhaite que la tâche soit finie). Le respect de la priorité à un impact sur le respect des échéances, ce qui nous conduit à appliquer des mécanismes de fallback. Notons que nos algorithmes seront évalués par simulations.

Mots-clés : Ordonnancement, Environnement de résolution de problèmes 


\section{Introduction}

The Grid provides a solution to compute huge applications over the Internet [7]. Several approaches co-exist like object-oriented languages, message passing environments, infrastructure toolkits, Web-based, and global computing environments. The Remote Procedure Call (RPC) [9] paradigm seems also to be a good candidate to build Problem Solving Environments (PSE) for different applications on the Grid. Indeed, it allows one to hide complicated resource allocation and job distribution strategies from the user. Moreover, parallelism can be hidden using parallel servers. Several environments following this approach exist, like NetSolve [1], Ninf [10] or DIET [2]. They are commonly called Network Enabled Server (NES) environments [9].

Currently, NES systems use the Minimum Completion Time (MCT) on-line scheduling algorithm. The deadline scheduling model uses the expected response time of each execution as a scheduling parameter called a "deadline". We assume that each task, when it arrives in the scheduler, has a given static deadline (possibly given by the client). If a task completes its execution before its chosen relative deadline, it meets the deadline. Otherwise the tasks fails.

We divide our contribution in two parts. The main idea of the first part is to introduce the mechanism of performance prediction for tasks. The aim of the second part is to give an expanded version of deadline scheduling with priority mechanism. The presented scheduling algorithms aim at scheduling sequential tasks on a NES environment. This kind of environment is usually composed of an agent receiving tasks and finding the most efficient server that will be able to execute a given task on behalf of the client.

\section{Related Work}

While a large number of papers describe priority algorithms for classical operating systems, little research exists around scheduling algorithms using priority and deadline for grid applications.

Scheduling problems that combine tails and deadlines is discussed in [12]. Lower bounds are given for the shop scheduling problems and an algorithm is presented with an improved complexity to solve two parallel machine problem with unit execution time operations. An algorithm in [14] finds minimum-lateness schedules for different classes of DAGS when each task has to be executed in a non-uniform interval. Both papers present interesting theoretical results that can not be directly implemented in a grid platform due to some limitations of models. 
A deadline scheduling strategy is given in [13] for multi-client multi-server case on a grid platform. The authors assume that each task (tasks sent to the scheduling agent) receive a deadline from the client. The algorithm presented aims at minimizing deadline misses. It is also augmented with "Load Correction" and "fallback" mechanisms to improve its performance. The first optimization is done by taking into account load changes (due to previous scheduling decisions) as soon as possible. The fallback mechanisms makes some corrections to the schedule at the server level. If the server finds that a task will not meet the deadline due to prediction errors, the task is re-submitted to the system. Simulation has been provided using the Bricks simulation framework. A background load is simulated and real traces are injected in the model to simulate the extra load of the system. Optimized algorithms are compared to a simple greedy algorithm that does not take deadlines into account. This algorithm is indeed less efficient than the optimized ones. The simulation shows that while the load correction mechanism does not improve the overall execution time significantly, the fallback optimization leads to important reductions of the failure rates without increasing the cost of scheduling.

\section{FAST's Overview}

Our first contribution consist to do performance prediction using a forecasting tool Fast Agent's System Timer ( FAST ) [5, 11]. FAST is a dynamic performance forecasting tool for Grid environments designed to handle these important issues. Information acquired by FAST concerns sequential computation routines and their execution platforms. For example it can be useful to a scheduler to optimize task mapping.

As shown in Figure 1, FAST is composed of two mainmodules offering a user API: the static data acquisition module and the dynamic data acquisition module. The former model forecasts the time and space needs of a sequential computation routine on a given machine for a given set of parameters, while the latter forecasts the behavior of dynamically changing resources, e.g., workload, bandwidth or memory use. FAST relies on low-level software packages. First, LDAP [8] is used to store static data. Then to acquire dynamic data. FAST is essentially based on the NWS (Network Weather Service) [16], a project initiated at the University of California San Diego and now being developed at the University of Santa Barbara. It is a distributed system that periodically monitors and dynamically forecasts performance of various network and computational resources. NWS can monitor several resources including communications links, CPU, disk space, and memory. Moreover, NWS is not only able to obtain measurements, it can also forecast the evolution of the monitored system. 
FAST extends NWS as it allows to determine theoretical needs of computation routines in terms of execution time and memory. The current version of FAST only handles regular sequential routines like those of the dense linear algebra library BLAS [6]. However BLAS kernels represent the heart of many applications, especially in numerical simulation. The approach chosen by FAST to forecast execution times of such routines is an extensive benchmark followed by a polynomial regression. Indeed this kind of routines is often strongly optimized with regard to the platform, either by vendors or by automatic code generation [15]. FAST is also able to forecast the use of parallel routines [3]. However, only the sequential part of FAST will be used in this article.

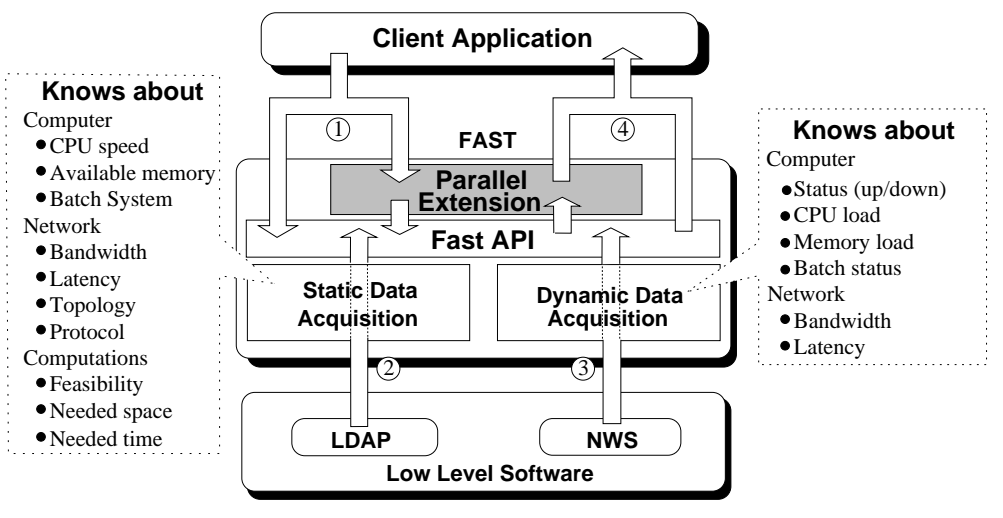

Figure 1: Overview of the FAST architecture.

In this paper FAST and NWS will be used to give the availability of CPU and network, and to know the execution time of a specific task on a specific server.

\section{Client-Server Scheduling algorithms}

\subsection{Client-Server Scheduler with load measurements}

As we target heterogeneous architectures, each task can have a different execution time on each server. Let $T_{a S_{i}}$ be the execution time for the task $a$ on server $i$. This time includes the time to send the data, the time to receive the result of the computation and the execution time:

$$
T_{a S_{i}}=\frac{W_{\text {send }}}{P_{\text {send }}}+\frac{W_{\text {recv }}}{P_{\text {recv }}}+\frac{W_{a S_{i}}}{P_{S}}
$$


where $W_{\text {send }}$ is the size of the data transmitted from the client to the server, $W_{\text {recv }}$ denotes to the data size transmitted from the server to the client, $W_{a S_{i}}$ is the number of floating point operations of the task, $P_{\text {send }}$ denotes the predicted network throughputs from the client to the server, $P_{\text {recv }}$ denotes the predicted network throughputs from the server to the client and $P_{S}$ is the server performance (in floating point operations per second).

$P_{\text {send }}$ should be replaced by the network throughput value measured just before the task. This value is returned by one call to FAST (cf. Section 3). $P_{\text {recv }}$ is estimated using previous measurements. The CPU performance is also dynamic and depends on other tasks running on the target processors. Thus, FAST can be used to provide a forecast of CPU performance, so as to take into account the actual CPU workload.

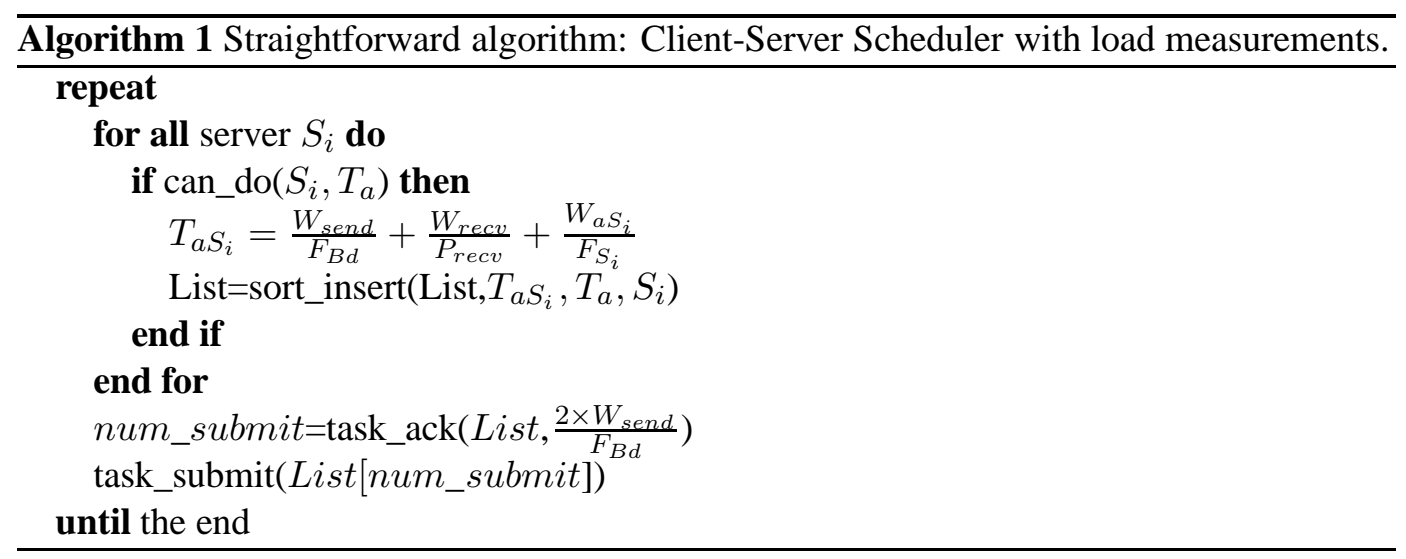

Algorithm 1 gives a straightforward algorithm to get a sorted list of servers that are able to compute the client's task. It assumes that the client takes the first available server, which is most efficient, from the list. However, a loop can be added at the end of the algorithm between the task_ack and task_submit calls.

For sake of simplicity we define four functions for Algorithm 1:

can_do This function returns true if server $S_{i}$ have the resource required to compute task $T_{a}$. This function takes into account the availability of memory and disk storage, the computational library etc.

sort_insert This function sorts servers by efficiency. As an input, we have the current List of servers, the time predicted $T_{a S_{i}}$, the task name $T_{a}$ and the server name $S_{i}$. Its output is the List of ordered servers.

task_ack This function sends the data and the computation task. To avoid a dead-lock due to network problems, the function chooses the next server in the list if the time 
to send the data is greater than the time given in the second parameter. The output of this function is the server where the data are sent (index number in the array List).

task_submit This function performs the remote execution on the server given by task_ack.

\subsection{Client-Server Scheduler with a Forecast Correction Mechanism}

The previous algorithm assumes that FAST always returns an accurate forecast. In this section, we take into account the gap between the performance prediction and the actual execution time of each task.

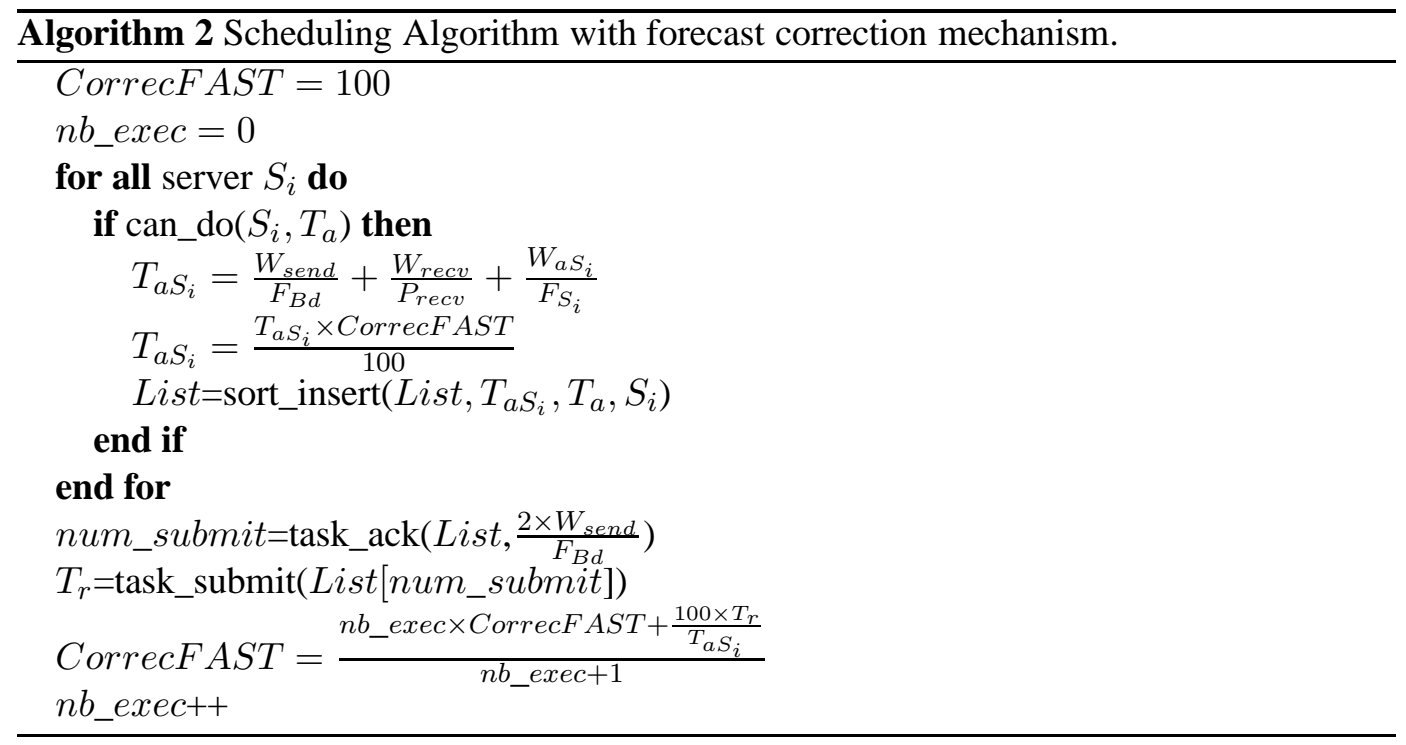

The function task_submit is upgraded to return the time of the remote execution. Thus, we can modify the next predictions from the monitoring system as follows to obtain the corrected load values:

$$
T_{a S_{i}}=\frac{T_{a S_{i}} \times \text { CorrecFAST }}{100}
$$

where CorrecFAST is an error average between the prediction time and the actual execution time. This value is updated at each execution as follows:

$$
\text { CorrecFAST }=\frac{n b \_e x e c \times C o r r e c F A S T+\frac{100 \times T_{r}}{T_{a S_{i}}}}{n b \_e x e c+1}
$$


where $T_{r}$ is the actual execution time and $T_{a S_{i}}$ the time predicted. Algorithm 2 includes this correction mechanism.

\subsection{Client-Server Scheduler with a Priority Mechanism}

Until now, for client-server system, either the deadline scheduling or the priority based scheduling are considered. Here we give an algorithm that utilizes both criteria to select a server for the task.

In Algorithm 3, tasks have a predefined priority and deadline. A task is allocated on the server task queue according to its priority. If a task can meet its deadline then it is sent to the server for execution. For Algorithm 3, we define some new variables. $T D_{a}$ is the deadline and $T P_{a}$ is the priority of task $T_{a} . T F_{a S_{i}}$ is the changed execution time of task $T_{a}$ on server $S_{i}$ after placing it on the server task queue. To simplify the explanation of the algorithm, we define five functions:

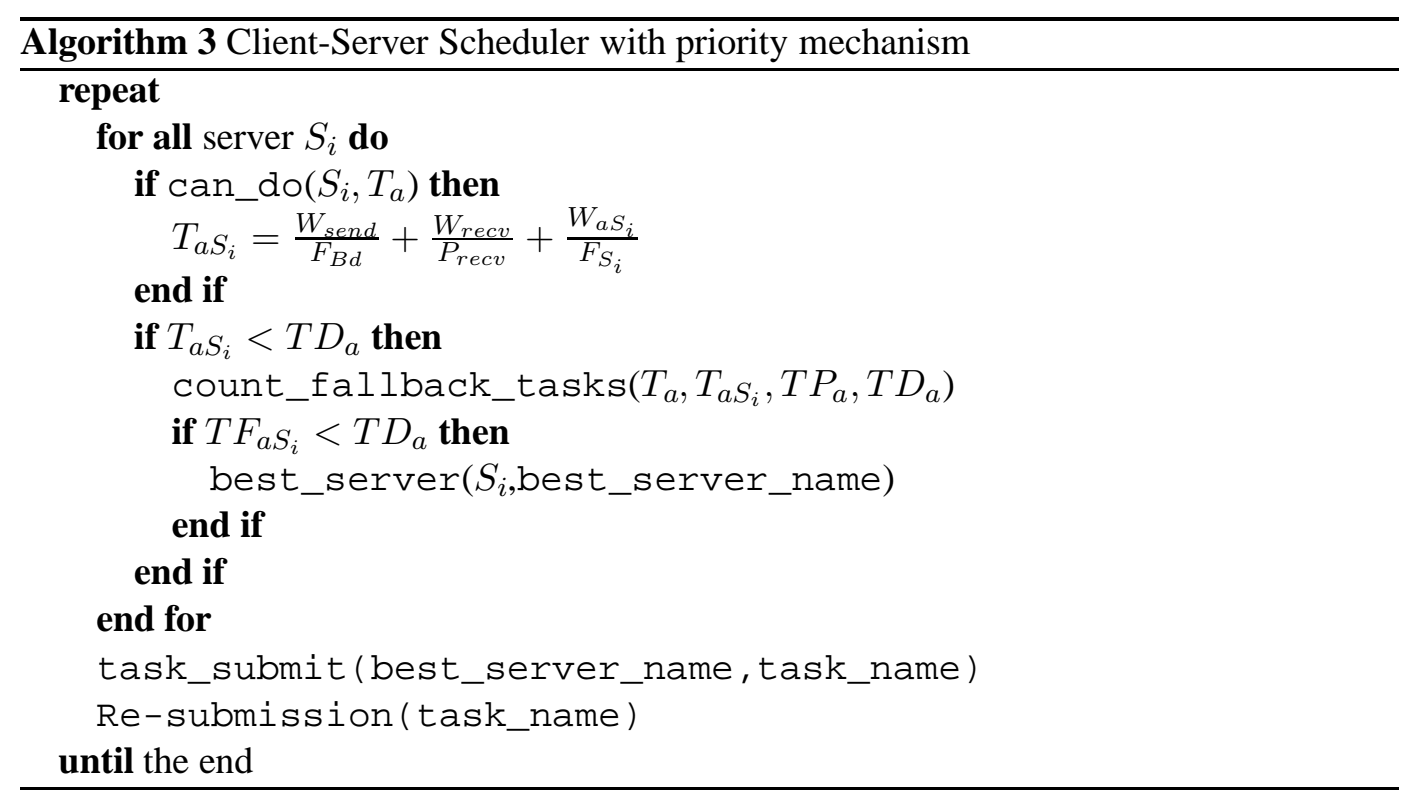

can_do This function works like for Algorithm 1.

Count_fallback_tasks This function counts the fallbacked tasks. Tasks that cannot meet their deadline after the insertion of the new task are called fallbacked tasks. Task $T_{a}$ is placed according to its priority $T P_{a}$ on the server task queue, which may change the execution time of the tasks on the queue. 
best_server This function selects the best server among the servers that can execute the task within the deadline time. The best server is selected by comparing the number of fallbacked tasks. Server with less fallbacked tasks is selected for the task execution. If the servers have same number of fallbacked tasks, then the time to compute the task is compared and the server that takes less time is selected.

task_submit This function works the like in Algorithm 1. It performs the remote execution on the server given by the best_server but in this case the argument of the function one server and not a list of servers.

Re-submission This function submits the fallbacked task to the servers, for recomputing the execution time. If any server can meet the task's deadline then the task is allocated to that server.

\begin{tabular}{|c|c|c|c|c|c|}
\cline { 4 - 6 } \multicolumn{2}{c|}{} & \multicolumn{3}{c|}{$\begin{array}{c}\text { Exec. time } \\
\text { on server }\end{array}$} \\
\hline Task & Priority & Deadline & $S_{1}$ & $S_{2}$ & $S_{3}$ \\
\hline 1 & 3 & 15 & 3 & 5 & 6 \\
\hline 2 & 5 & 10 & 5 & 12 & 9 \\
\hline 3 & 2 & 30 & 11 & 20 & 15 \\
\hline 4 & 4 & 20 & 10 & np & 17 \\
\hline 5 & 5 & 15 & 12 & 14 & np \\
\hline
\end{tabular}

Table 1: Priority, deadline and computation time of each task.

Figure 2 shows an example to explain the behavior of Algorithm 3. Lets consider 3 servers with different capacities and 5 tasks. The priority, deadline, and computation time of each task on each server is shown in Table 1. Here computation time is the time taken by the dedicated server to compute the task when the server is free. Computation value $n p$ denotes that the task cannot be executed on the server, which maybe due to the type of the task, memory requirement etc. A task is allocated to the server while checking the execution time on each server, its priority and deadline. Task $T_{1}$ is allocated to server $S_{1}$. Task $T_{2}$ is also allocated to server $S_{1}$. As its priority is higher it shifts the task $T_{1}$, so the execution time of $T_{1}$ is changed. If task $T_{3}$ is placed on server $S_{1}$, it will take less execution time but due to its priority the execution time will be changed. So task $T_{3}$ is placed on server $S_{3}$. Task $T_{4}$ is placed on server $S_{1}$, but while doing so task $T_{1}$ is fallbacked. Re-submission of task $T_{1}$ is done and it is allocated to server $S_{2}$. Task $T_{5}$ is placed on server $S_{2}$. As its 

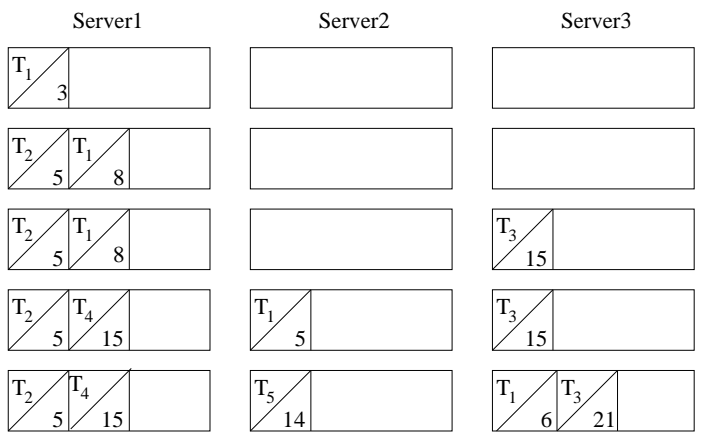

Figure 2: Example for priority scheduling algorithm with fallback mechanism. Task id and execution time is written diagonally in each box.

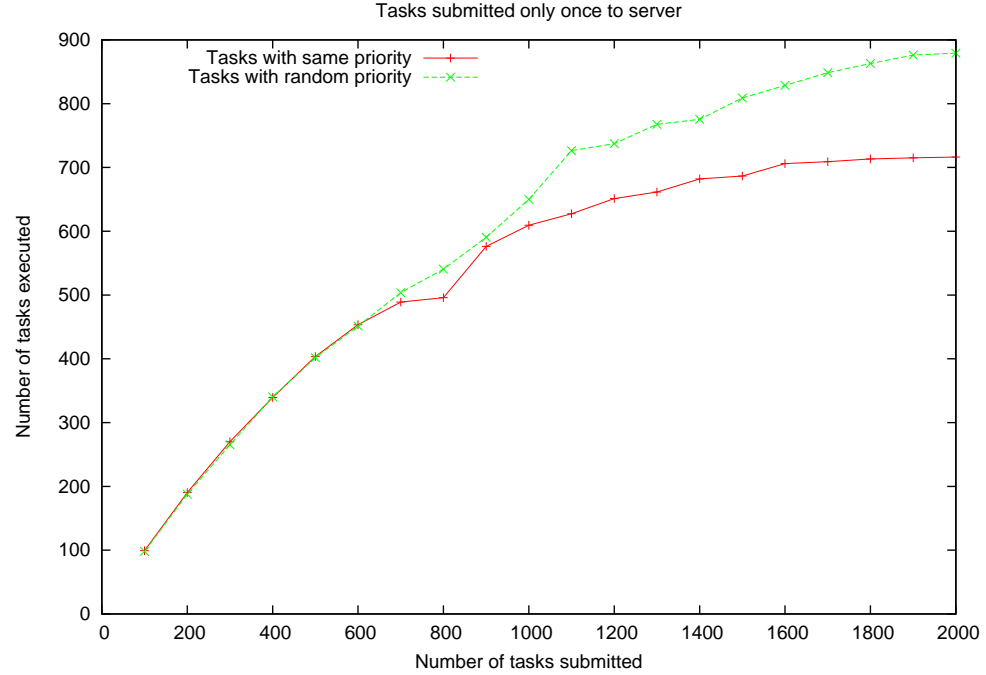

Figure 3: Priority based tasks are executed without fallback mechanism.

priority is higher than task $T_{1}$, execution time of $T_{1}$ changed and the task is then fallbacked. Again re-submission is done and task $T_{1}$ is placed on server $S_{3}$. 


\section{Simulation Results}

To simulate the deadline algorithm with priority and the impact of fallback mechanism with this model we use a simulation toolkit called Simgrid [4]. Simgrid provides an excellent framework for setting up a simulation where decisions are taken by a single scheduling process.

We took 100 servers to execute the tasks in our experiments. Each task is associated with a priority and deadline. We randomly generated the priority between 1 and 10 and considered tasks deadline to be 5 times of the computation amount needed by the task on dedicated server.

We did experiments by fixing the priority of the tasks and varying the priority depending on tasks' size. Figure 3 shows when tasks with same priority is submitted the number of executed tasks is less than the tasks executed with random priority. When the number of tasks is less the impact of task priority is negligible. But as the number of tasks increases, tasks' priority plays an important role for increasing the number of tasks executed.

We used Algorithm 3 to check the impact of fallback mechanism on the number of tasks executed under different criteria in Figure 4. Figure 4(a) shows that the fallback mechanism has no effect if the tasks have the same priority. But in Figure 4(b), 4(c), and 4(d), it can be seen that the fallback mechanism is very useful as the number of submitted tasks (with priority and different sizes) is increased.

\section{Conclusions and Future Work}

We have presented algorithms for scheduling of sequential tasks for client-server systems on the grid. This article is the an extension of the paper: "A Study of Deadline Scheduling for Client-Server Systems on the Computational Grid" [13]. We gave the introduction of the performance prediction tool FAST $[5,11]$ and mainly focused on the management of tasks with respect to their priorities and deadlines. Load correction mechanism using FAST and fallback mechanism are used to increase the number of executed tasks. We presented an algorithm that considers both priority and deadline of the tasks to select a server. We showed through experiments that the number of tasks that can meet their deadlines can be increased by 1) using task priorities and by 2) using a fallback mechanism to reschedule tasks that were not able to meet their deadline on the selected servers.

Our future work consists in implementing these scheduling algorithms in the DIET platform [2]. 


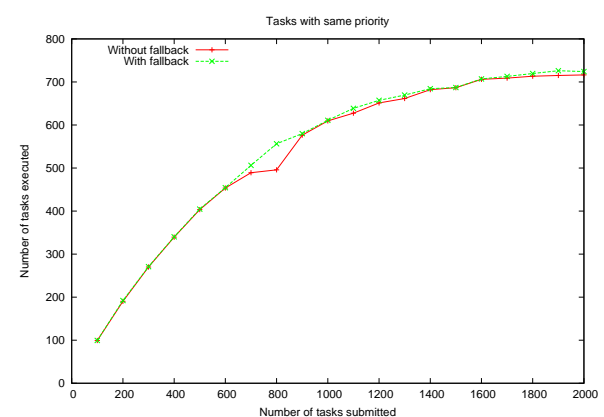

(a) Tasks without priority

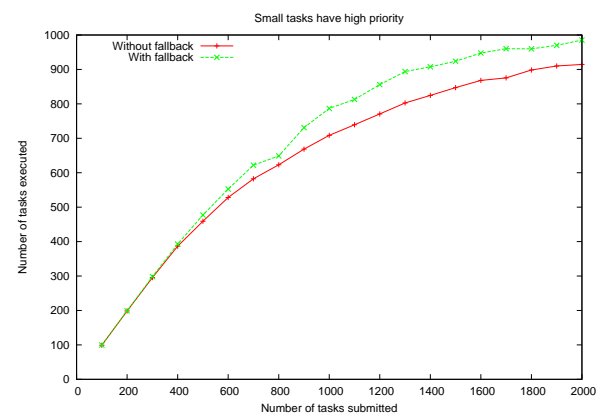

(c) Tasks with execution time less than 15 minutes on dedicated servers

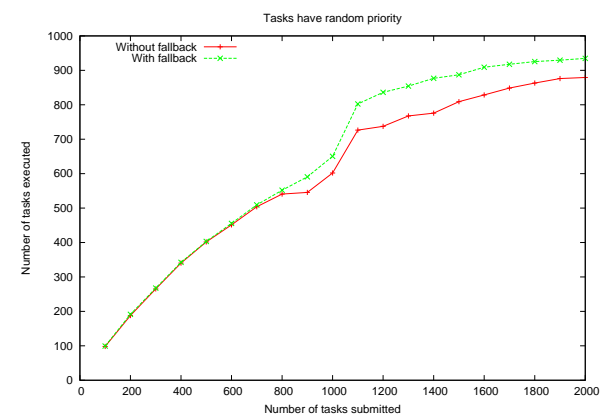

(b) Tasks priority vary between 1-10

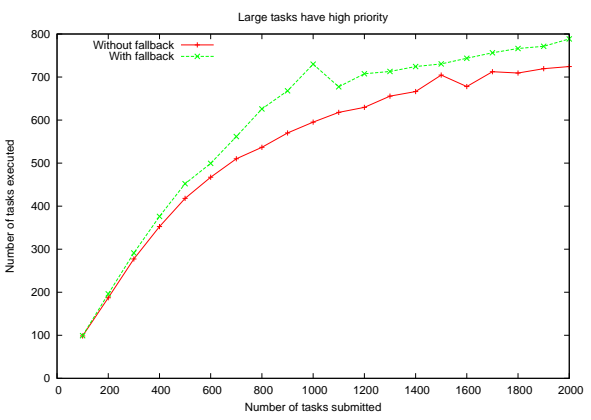

(d) Tasks with execution time greater than 4 hours on dedicated server

Figure 4: Comparison of tasks executed with and without fallback.

\section{References}

[1] D. Arnold, S. Agrawal, S. Blackford, J. Dongarra, M. Miller, K. Sagi, Z. Shi, and S. Vadhiyar. Users' Guide to NetSolve V1.4. Computer Science Dept. Technical Report CS-01467, University of Tennessee, Knoxville, TN, July 2001. http://www.cs.utk .edu/ netsolve/.

[2] E. Caron, F. Desprez, F. Lombard, J.-M. Nicod, M. Quinson, and F. Suter. A Scalable Approach to Network Enabled Servers. In Proceedings of EuroPar 2002, Paderborn, Germany, 2002.

[3] E. Caron and F. Suter. Parallel Extension of a Dynamic Performance Forecasting Tool. In Proceedings of the International Symposium on Parallel and Distributed Computing, pages 
80-93, Iasi, Romania, Jul 2002.

[4] H. Casanova. SIMGRID: A toolkit for the simulation of application scheduling. In Proceedings of the IEEE Symposium on Cluster Computing and the Grid (CCGrid'01). IEEE Computer Society, May 2001.

[5] F. Desprez, M. Quinson, and F. Suter. Dynamic Performance Forecasting for Network Enabled Servers in a Heterogeneous Environment. In Proceedings of the International Conference on Parallel and Distributed Processing Techniques and Applications (PDPTA), June 2001.

[6] J. Dongarra, J. Du Croz, S. Hammarling, and R. Hanson. An Extended Set of Fortran Basic Linear Algebra Subroutines. 14(1):1-17, 1988.

[7] I. Foster and C. K. (Eds.). The Grid: Blueprint for a New Computing Infrastructure. MorganKaufmann, 1998.

[8] T. Howes, M. Smith, and G. Good. Understanding and deploying LDAP directory services. Macmillian Technical Publishing, 1999. ISBN: 1-57870-070-1.

[9] S. Matsuoka, H. Nakada, M. Sato, and S. Sekiguchi. Design Issues of Network Enabled Server Systems for the Grid. http://www.eece.unm.edu/ dbader/grid/ WhitePapers/satoshi.pdf, 2000. Grid Forum, Advanced Programming Models Working Group whitepaper.

[10] H. Nakada, M. Sato, and S. Sekiguchi. Design and Implementations of Ninf: towards a Global Computing Infrastructure. Future Generation Computing Systems, Metacomputing Issue, 15(5-6):649-658, 1999. http://ninf.apgrid.org/papers/papers.shtml.

[11] M. Quinson. Dynamic Performance Forecasting for Network-Enabled Servers in a Metacomputing Environment. In Proceedings of the International Workshop on Performance Modeling, Evaluation, and Optimization of Parallel and Distributed Systems (PMEO-PDS'02), Apr 2002.

[12] F. Sourd and W. Nuijten. Scheduling with Tails and Dead-lines. Journal of Scheduling, 4:105$121,2001$.

[13] A. Takefusa, H. Casanova, S. Matsuoka, and F. Berman. A study of deadline scheduling for client-server systems on the computational grid. In the 10th IEEE Symposium on High Performance and Distributed Computing (HPDC'01), San Francisco, California., Aug. 2001.

[14] J. Verriet. Scheduling UET, UCT DAGS with Release Dates and Deadlines. Technical report, Utrecht University, Department of Computer Science, 1995.

[15] R. Whaley and J. Dongarra. Automatically Tuned Linear Algebra Software (ATLAS). In Proceedings of IEEE SC'98, 1998. Best Paper award.

[16] R. Wolski, N. Spring, and J. Hayes. The Network Weather Service: A Distributed Resource Performance Forecasting Service for Metacomputing. Future Generation Computing Systems, Metacomputing Issue, 15(5-6):757-768, Oct. 1999. 
Unité de recherche INRIA Rhône-Alpes 655, avenue de l'Europe - 38334 Montbonnot Saint-Ismier (France)

Unité de recherche INRIA Futurs : Parc Club Orsay Université - ZAC des Vignes 4, rue Jacques Monod - 91893 ORSAY Cedex (France) Unité de recherche INRIA Lorraine : LORIA, Technopôle de Nancy-Brabois - Campus scientifi que 615, rue du Jardin Botanique - BP 101 - 54602 Villers-lès-Nancy Cedex (France)

Unité de recherche INRIA Rennes : IRISA, Campus universitaire de Beaulieu - 35042 Rennes Cedex (France) Unité de recherche INRIA Rocquencourt : Domaine de Voluceau - Rocquencourt - BP 105 - 78153 Le Chesnay Cedex (France) Unité de recherche INRIA Sophia Antipolis : 2004, route des Lucioles - BP 93 - 06902 Sophia Antipolis Cedex (France) 